ANNALES

POLONICI MATHEMATICI

$85.2(2005)$

\title{
Criteria for univalence, starlikeness and convexity
}

\author{
by S. Ponnusamy and P. Vasundhra (Chennai)
}

Abstract. Let $\mathcal{A}$ denote the class of all normalized analytic functions $f(f(0)=0=$ $\left.f^{\prime}(0)-1\right)$ in the open unit disc $\Delta$. For $0<\lambda \leq 1$, define

and

$$
\mathcal{U}(\lambda)=\left\{f \in \mathcal{A}:\left|\left(\frac{z}{f(z)}\right)^{2} f^{\prime}(z)-1\right|<\lambda, z \in \Delta\right\}
$$

$$
\mathcal{P}(2 \lambda)=\left\{f \in \mathcal{A}:\left|\left(\frac{z}{f(z)}\right)^{\prime \prime}\right|<2 \lambda, z \in \Delta\right\} .
$$

Recently, the problem of finding the starlikeness of these classes has been considered by Obradović and Ponnusamy, and later by Obradović et al. In this paper, the authors consider the problem of finding the order of starlikeness and of convexity of $\mathcal{U}(\lambda)$ and $\mathcal{P}(2 \lambda)$, respectively. In particular, for $f \in \mathcal{A}$ with $f^{\prime \prime}(0)=0$, we find conditions on $\lambda$, $\beta^{*}(\lambda)$ and $\beta(\lambda)$ so that $\mathcal{U}(\lambda) \subsetneq \mathcal{S}^{*}\left(\beta^{*}(\lambda)\right)$ and $\mathcal{P}(2 \lambda) \subsetneq \mathcal{K}(\beta(\lambda))$. Here, $\mathcal{S}^{*}(\beta)$ and $\mathcal{K}(\beta)$ $(\beta<1)$ denote the classes of functions in $\mathcal{A}$ that are starlike of order $\beta$ and convex of order $\beta$, respectively. In addition to these results, we also provide a coefficient condition for functions to be in $\mathcal{K}(\beta)$. Finally, we propose a conjecture that each function $f \in \mathcal{U}(\lambda)$ with $f^{\prime \prime}(0)=0$ is convex at least when $0<\lambda \leq 3-2 \sqrt{2}$.

1. Introduction and main results. Let $\mathcal{A}$ be the class of analytic functions $f$ in the open unit disk $\Delta=\{z \in \mathbb{C}:|z|<1\}$ with the normalization $f(0)=0=f^{\prime}(0)-1$. The subclass of $\mathcal{A}$ consisting of univalent functions is denoted by $\mathcal{S}$. Several subclasses of univalent functions play a prominent role in the theory of univalent functions $[1,2]$. Among them are the class of all convex functions of order $\beta, \beta<1$, given by

$$
\mathcal{K}(\beta)=\left\{f \in \mathcal{A}: \operatorname{Re}\left(z f^{\prime \prime}(z) / f^{\prime}(z)+1\right)>\beta, z \in \Delta\right\}
$$

and the class of all starlike functions of order $\beta, \beta<1$, described by

$$
\mathcal{S}^{*}(\beta)=\left\{f \in \mathcal{A}: \operatorname{Re}\left(z f^{\prime}(z) / f(z)\right)>\beta, z \in \Delta\right\} .
$$

2000 Mathematics Subject Classification: 30A10, 30C45, 30C55, 30C80.

Key words and phrases: univalent, starlike and convex functions.

The work of the authors was supported by National Board for Higher Mathematics (Grant No. 48/1/98-R\&D-II/18 through NBHM/DAE, India). The authors thank Dr. G. P. Youvaraj for his help in the proof of Theorem 1.2.

Also, the authors thank the referee for his/her valuable comments which, in particular, simplified the proof of Theorem 1.2. 
The class of all convex functions denoted by $\mathcal{K}:=\mathcal{K}(0)$ consists of all $f \in \mathcal{S}$ for which the range $f(\Delta)$ is convex. Similarly, the class of starlike functions denoted by $\mathcal{S}^{*}:=\mathcal{S}^{*}(0)$ consists of all $f \in \mathcal{S}$ for which $f(\Delta)$ is starlike (with respect to the origin). An important member of the class $\mathcal{S}$ is the Koebe function $k(z)=z /(1-z)^{2}$ together with its rotations. An interesting subclass of $\mathcal{S}$ containing the Koebe function is the class $\mathcal{U}:=\mathcal{U}(1)$, where $\mathcal{U}(\lambda)(0<\lambda \leq 1)$ is defined by

$$
\mathcal{U}(\lambda)=\left\{f \in \mathcal{A}:\left|\left(\frac{z}{f(z)}\right)^{2} f^{\prime}(z)-1\right|<\lambda, z \in \Delta\right\} .
$$

This class has been studied by Obradović and Ponnusamy [3] together with the class

$$
\mathcal{P}(2 \lambda)=\left\{f \in \mathcal{A}:\left|\left(\frac{z}{f(z)}\right)^{\prime \prime}\right|<2 \lambda, z \in \Delta\right\} .
$$

There are many relationships between various subclasses of $\mathcal{S}$. However, the classes $\mathcal{U}(1)$ and its direct generalization $\mathcal{U}(\lambda)$ have not been looked at until recently. According to a result due to Ozaki and Nunokawa [6], we have the inclusion

$$
\mathcal{U}(\lambda) \subset \mathcal{S} \quad \text { for } 0<\lambda \leq 1,
$$

and from [3], we also have the inclusion $\mathcal{P}(2 \lambda) \subset \mathcal{U}(\lambda)$. In [4], the authors have shown that certain results obtained in [3] also hold if $\mathcal{P}(2 \lambda)$ is replaced by $\mathcal{U}(\lambda)$. In this connection, we recall the following result from [4].

Theorem 1.1. If $f(z)=z+\sum_{n=2}^{\infty} a_{n} z^{n} \in \mathcal{U}(\lambda)$, then $f \in \mathcal{S}^{*}$ for $0<\lambda \leq \lambda^{*}$, where

$$
\lambda^{*}=\frac{-a+\sqrt{2-a^{2}}}{2} \quad \text { with } \quad a=\left|f^{\prime \prime}(0)\right| / 2 .
$$

This result was originally stated as a conjecture in [3] and was proved in [4]. In this article, we discuss the relationship between $\mathcal{U}(\lambda)$ and $\mathcal{S}^{*}(\delta)$, as well as between $\mathcal{P}(2 \lambda)$ and $\mathcal{K}(\delta)$. As a consequence, we improve certain coefficient results due to Reade, Silverman and Todorov [7].

We now state our first result which gives a condition for functions in $\mathcal{U}(\lambda)$ to be starlike of order $\delta(\lambda)$.

THEOREM 1.2. If $f \in \mathcal{U}(\lambda)$ and $a=\left|f^{\prime \prime}(0)\right| / 2 \leq 1$, then $f \in \mathcal{S}^{*}(\delta)$ whenever $0<\lambda \leq \lambda(\delta)$, where

$$
\lambda(\delta)= \begin{cases}\frac{\sqrt{(1-2 \delta)\left(2-a^{2}-2 \delta\right)}-a(1-2 \delta)}{2(1-\delta)} & \text { if } 0 \leq \delta<\frac{1+a}{3+a}, \\ \frac{1-\delta(1+a)}{1+\delta} & \text { if } \frac{1+a}{3+a} \leq \delta<\frac{1}{1+a} .\end{cases}
$$


We observe that if we choose $\delta=0$ in Theorem 1.2, then Theorem 1.1 follows. Also, we note that

$$
\lambda\left(\frac{1-a^{2}}{2}\right)= \begin{cases}\frac{1-a+a^{2}+a^{3}}{3-a^{2}} & \text { if } 0 \leq a \leq \sqrt{2}-1, \\ \frac{a\left(1-a^{2}\right)}{1+a^{2}} & \text { if } \sqrt{2}-1 \leq a<1 .\end{cases}
$$

Therefore, Theorem 1.2 is an extension of Theorem 1.1. Further, we believe that the order of starlikeness given above for functions in $\mathcal{U}(\lambda)$ is sharp although at present we do not have a concrete proof. However, from Theorem 1.2, one can obtain a number of new results. For example, if $f \in \mathcal{U}(\lambda)$ and $a=\left|f^{\prime \prime}(0) / 2\right|<1$ then $f \in \mathcal{S}^{*}(1 / 2)$ whenever $0<\lambda \leq(1-a) / 3$.

Corollary 1.3. If $f \in \mathcal{U}(\lambda)$ with $f^{\prime \prime}(0)=0$, then $f \in \mathcal{S}^{*}(\delta)$ whenever

$$
0<\lambda \leq \lambda(\delta)= \begin{cases}\sqrt{\frac{1-2 \delta}{2(1-\delta)}} & \text { if } 0 \leq \delta \leq 1 / 3, \\ \frac{1-\delta}{1+\delta} & \text { if } 1 / 3 \leq \delta<1,\end{cases}
$$

or equivalently

$$
\delta:=\delta(\lambda)= \begin{cases}\frac{1-\lambda}{1+\lambda} & \text { if } 0<\lambda \leq 1 / 2 \\ \frac{1-2 \lambda^{2}}{2\left(1-\lambda^{2}\right)} & \text { if } 1 / 2 \leq \lambda \leq 1 / \sqrt{2} .\end{cases}
$$

In particular,

- $f \in \mathcal{U}(\lambda), f^{\prime \prime}(0)=0$ and $0<\lambda \leq 1 / \sqrt{2} \Rightarrow f \in \mathcal{S}^{*}$,

- $f \in \mathcal{U}(\lambda), f^{\prime \prime}(0)=0$ and $0<\lambda \leq 1 / 3 \Rightarrow f \in \mathcal{S}^{*}(1 / 2)$.

Our next result provides an affirmative answer to the following

Problem 1.4. Find conditions on $\lambda$ and $\beta(\lambda)$ so that $\mathcal{P}(2 \lambda) \subset \mathcal{K}(\beta(\lambda))$.

We recall that $\mathcal{P}(2 \lambda) \subset \mathcal{S}^{*}$ if $0<\lambda \leq\left(-a+\sqrt{2-a^{2}}\right) / 2, a=\left|f^{\prime \prime}(0)\right| / 2$.

Theorem 1.5. Let $f \in \mathcal{A}$ with $f^{\prime \prime}(0)=0$ and suppose that

$$
\left|\left(\frac{z}{f(z)}\right)^{2} f^{\prime}(z)\left(1+\frac{z f^{\prime \prime}(z)}{2 f^{\prime}(z)}-\frac{z f^{\prime}(z)}{f(z)}\right)\right|<\lambda, \quad z \in \Delta,
$$

or equivalently,

$$
\left|z^{2}\left(\frac{z}{f(z)}\right)^{\prime \prime}\right|<2 \lambda, \quad z \in \Delta
$$


for some $0<\lambda \leq 1 / \sqrt{2}$. Then $f \in \mathcal{K}(\beta)$, where

$$
\beta=\beta(\lambda)= \begin{cases}\frac{1+\lambda^{2}-6 \lambda}{1-\lambda^{2}} & \text { for } 0<\lambda \leq 1 / 2, \\ \frac{-\lambda(2+3 \lambda)}{1-\lambda^{2}} & \text { for } 1 / 2<\lambda \leq 1 / \sqrt{2} .\end{cases}
$$

In particular, $\mathcal{P}(2 \lambda) \subset \mathcal{K}$ if $0<\lambda \leq 3-2 \sqrt{2}$.

For our next result, we consider functions $f$ in $\mathcal{A}$ of the form

$$
f(z)=\frac{z}{\phi(z)},
$$

where $\phi(z)=1+\sum_{k=1}^{\infty} b_{k} z^{k}$ has no zeros in $\Delta$. Functions of this form have been studied, for example, in [7, 3]. In [3], Obradović and Ponnusamy obtained various coefficient conditions in terms of $b_{k}$ 's for the corresponding $f$ of the above form to be univalent, strongly starlike etc. In particular, the following results are known:

TheOREM 1.6. A function of the form (1.4) is in $\mathcal{K}$ if any one of the following conditions holds:

(i) there exist $p, q>0$ with $1 / p+1 / q \leq 1$ such that

$$
\begin{array}{r}
(2 p+1)\left|b_{1}\right|+\max \left\{\sum_{k=1}^{\infty}(2 k p+1)\left|b_{k}\right|, \sum_{k=1}^{\infty}(k-1)(k q+1)\left|b_{k}\right|\right\} \leq 1 . \\
\text { (ii) } \sum_{k=2}^{\infty}(k-1) k\left|b_{k}\right| \leq 2 \lambda, \quad \text { where } \lambda=\frac{7+\left|b_{1}\right|-\sqrt{33+30\left|b_{1}\right|+\left|b_{1}\right|^{2}}}{8} .
\end{array}
$$

Theorem 1.6(i) is due to [7] while Theorem 1.6(ii) has been obtained recently by Obradović et al. [4]. Our next result improves Theorem 1.6.

Theorem 1.7. Let $0<\lambda \leq 1 / \sqrt{2}$. If $f \in \mathcal{A}$ is of the form (1.4) and satisfies the coefficient condition

$$
\sum_{k=2}^{\infty}(k-1) k\left|b_{k}\right| \leq 2 \lambda,
$$

then $f \in \mathcal{K}(\beta)$, where $\beta=\beta(\lambda)$ is defined by (1.3).

A comparison of the $\lambda$-values of Theorems 1.7 and 1.6 shows that Theorem 1.7 improves Theorem 1.6. Indeed, for the case $b_{1}=0$, it suffices to note that $(7-\sqrt{33}) / 8<3-2 \sqrt{2}$.

It would be an interesting problem to find the largest value of $\lambda$ so that (1.5) implies that $f$ defined by (1.4) is convex in $\Delta$.

We end this section with a result which provides a sufficient condition for a function $f$ to be starlike or univalent in $\Delta$. 
Theorem 1.8. Let $0 \leq \mu \leq 1,0<\lambda \leq 1$ and $f \in \mathcal{A}$.

(i) If $f$ satisfies

$$
\left|1+\frac{1}{\mu+1} \frac{z f^{\prime \prime}(z)}{f^{\prime}(z)}-\frac{z f^{\prime}(z)}{f(z)}\right|<\frac{\log (1+\lambda)}{\mu+1}, \quad z \in \Delta,
$$

then

$$
\left|\left(\frac{z}{f(z)}\right)^{\mu+1} f^{\prime}(z)-1\right|<\lambda, \quad z \in \Delta
$$

(ii) If $f$ is such that $f^{\prime \prime}(0)=0$ and satisfies the condition

$$
\left|1+\frac{1}{\mu+1} \frac{z f^{\prime \prime}(z)}{f^{\prime}(z)}-\frac{z f^{\prime}(z)}{f(z)}\right|<\frac{2 \log (1+\lambda)}{\mu+1}, \quad z \in \Delta,
$$

then

$$
\left|\left(\frac{z}{f(z)}\right)^{\mu+1} f^{\prime}(z)-1\right|<\lambda, \quad z \in \Delta .
$$

In [5, Theorem 2], Theorem 1.8(i) was proved with $\log (1+\lambda)$ in (1.6) replaced by $\lambda /(\lambda+1)$. Note that $\log (1+\lambda)>\lambda /(\lambda+1)$ for all $\lambda \in(0,1]$, so Theorem 1.8 improves the result of Obradović and Tuneski [5]. In particular, for each $0<\lambda \leq 1$ and $f \in \mathcal{A}$, one has

$$
\begin{gathered}
\left|1+\frac{z f^{\prime \prime}(z)}{f^{\prime}(z)}-\frac{z f^{\prime}(z)}{f(z)}\right|<\log (1+\lambda) \Rightarrow\left|\frac{z f^{\prime}(z)}{f(z)}-1\right|<\lambda, \quad z \in \Delta, \\
\left|\frac{z f^{\prime \prime}(z)}{f^{\prime}(z)}+2\left(1-\frac{z f^{\prime}(z)}{f(z)}\right)\right|<\log (1+\lambda) \Rightarrow\left|\left(\frac{z}{f(z)}\right)^{2} f^{\prime}(z)-1\right|<\lambda, \quad z \in \Delta .
\end{gathered}
$$

We observe that if $f^{\prime \prime}(0)=0$ then the last two implications hold with $2 \log (1+\lambda)$ in place of $\log (1+\lambda)$.

2. Proofs of the main theorems. For the proof of Theorem 1.5, we need a special case of the following lemma. However, from the proof of Theorem 1.5, we note that Lemma 2.1 may be used to state a more general result.

Lemma 2.1. Let $0<\lambda<1, \alpha>-2$ and let $g \in \mathcal{H}$, the class of all analytic functions in the unit disc $\Delta$, satisfy the condition $g(z) \prec 1+\lambda z$ for $z \in \Delta$ with $g(0)=1$. Suppose that $\operatorname{Re} \phi(z) \geq \delta$ in $\Delta$. If $p \in \mathcal{H}, p(0)=1$ and

$$
|g(z)(\beta+(1-\beta) p(z)+1-\alpha-2 \phi(z))-\alpha|<\lambda(\alpha+2), \quad z \in \Delta,
$$


where

$$
\beta=\left\{\begin{array}{l}
2 \delta+2 \alpha-\frac{1+\lambda}{1-\lambda} \quad \text { for }-2<\alpha \leq 2 \lambda \text { with } 0<\lambda<1, \\
2 \delta-1+\frac{2(\alpha-\lambda)}{1+\lambda} \quad \text { for } \alpha \geq \frac{2 \lambda}{1-2 \lambda} \text { with } 0<\lambda<1 / 2, \\
2 \delta+\frac{3 \alpha-2}{2}-\frac{(\alpha+2)^{2} \lambda^{2}}{2 \alpha\left(1-\lambda^{2}\right)} \\
\text { for } 2 \lambda \leq \alpha \leq \frac{2 \lambda}{1-2 \lambda} \text { with } 0<\lambda<1 / 2,
\end{array}\right.
$$

then $\operatorname{Re} p(z)>0$ for $z \in \Delta$.

Proof. In order to prove our result we notice that, in view of (2.1), it suffices to find $\inf _{|z|<1, \eta \in \mathbb{R}} \operatorname{Re} Q(z)$, where

$$
Q(z)=\frac{\alpha+\lambda(\alpha+2) z}{1+\lambda e^{i \eta} z}, \quad z \in \Delta .
$$

From (2.3) one can easily verify that

$$
\left|Q(z)-\frac{\alpha-r^{2} \lambda^{2}(\alpha+2) e^{-i \eta}}{1-r^{2} \lambda^{2}}\right| \leq \frac{r \lambda\left|\alpha-(\alpha+2) e^{i \eta}\right|}{1-r^{2} \lambda^{2}} \quad \text { for }|z| \leq r
$$

so that for $z \in \Delta$ we have

$$
\left|Q(z)-\frac{\alpha-\lambda^{2}(\alpha+2) e^{-i \eta}}{1-\lambda^{2}}\right| \leq \frac{\lambda\left|\alpha-(\alpha+2) e^{i \eta}\right|}{1-\lambda^{2}} .
$$

Therefore,

$$
\operatorname{Re} Q(z) \geq \frac{\alpha-\lambda^{2}(\alpha+2) \cos \eta-\lambda \sqrt{\alpha^{2}-2 \alpha(\alpha+2) \cos \eta+(\alpha+2)^{2}}}{1-\lambda^{2}} .
$$

Define $\psi(\eta)=\alpha-\lambda^{2}(\alpha+2) \cos \eta-\lambda \sqrt{\alpha^{2}-2 \alpha(\alpha+2) \cos \eta+(\alpha+2)^{2}}$.

CASE (i): If $-2<\alpha \leq 0$, then it is easy to see that $\psi$ is an increasing function on $[0, \pi]$ and therefore

$$
\psi(\eta) \geq \psi(0)=(1+\lambda)(\alpha-(\alpha+2) \lambda),
$$

which means that

$$
\operatorname{Re} Q(z) \geq \frac{\alpha-(\alpha+2) \lambda}{1-\lambda}, \quad z \in \Delta .
$$

CASE (ii): If $\alpha>0$ and $0<\lambda \leq \alpha / 2(\alpha+1)$, then we observe that $\psi$ is a decreasing function on $[0, \pi]$, which gives

$$
\psi(\eta) \geq \psi(\pi)=(\alpha-(\alpha+2) \lambda)(1-\lambda)
$$

and therefore,

$$
\operatorname{Re} Q(z) \geq \frac{\alpha-(\alpha+2) \lambda}{1+\lambda}, \quad z \in \Delta
$$


CASE (iii): Similarly, we find that

$$
\psi(\eta) \geq \psi\left(\eta_{1}\right)=\frac{\alpha^{2}-(\alpha+2)^{2} \lambda^{2}-\lambda^{2} \alpha^{2}}{2 \alpha}
$$

whenever $\lambda^{2}\left(\alpha^{2}-2 \alpha(\alpha+2) \cos \eta_{1}+(\alpha+2)^{2}\right)=\alpha^{2}$. This shows that

$$
\operatorname{Re} Q(z) \geq \frac{\alpha^{2}-(\alpha+2)^{2} \lambda^{2}-\lambda^{2} \alpha^{2}}{2 \alpha\left(1-\lambda^{2}\right)} \text { if } \frac{\alpha}{2(\alpha+1)} \leq \lambda \leq \frac{\alpha}{2} .
$$

CASE (iv): If $\lambda \geq \alpha / 2$, then we can easily see that $\psi$ is an increasing function on $[0, \pi]$ so that

$$
\psi(\eta) \geq \psi(0)=(\alpha-(\alpha+2) \lambda)(1+\lambda),
$$

which implies that

$$
\operatorname{Re} Q(z) \geq \frac{\alpha-(\alpha+2) \lambda}{1-\lambda}, \quad z \in \Delta .
$$

Finally, it follows that $\operatorname{Re} Q(z)>\beta_{0}(\alpha, \lambda), z \in \Delta$, where

$$
\beta_{0}(\alpha, \lambda)= \begin{cases}\frac{\alpha-(\alpha+2) \lambda}{1-\lambda} & \text { for }-2<\alpha \leq 2 \lambda \text { with } 0<\lambda<1, \\ \frac{\alpha-(\alpha+2) \lambda}{1+\lambda} & \text { for } \alpha \geq \frac{2 \lambda}{1-2 \lambda} \text { with } 0<\lambda<1 / 2, \\ \frac{\alpha^{2}\left(1-\lambda^{2}\right)-(\alpha+2)^{2} \lambda^{2}}{2 \alpha\left(1-\lambda^{2}\right)} & \text { for } 2 \lambda \leq \alpha \leq \frac{2 \lambda}{1-2 \lambda} \text { with } 0<\lambda<1 / 2 .\end{cases}
$$

A simple computation shows that $\beta=\beta_{0}(\alpha, \lambda)+2 \delta-(1-\alpha)$, where $\beta$ is given by (2.2). Therefore, $\operatorname{Re} Q(z)>\beta_{0}(\alpha, \lambda)$ is equivalent to

$$
\operatorname{Re} p(z)>\frac{\beta_{0}(\alpha, \lambda)+2 \delta-(1-\alpha)-\beta}{1-\beta}=0, \quad z \in \Delta,
$$

and the desired conclusion follows.

Proof of Theorem 1.2. Suppose that $f \in \mathcal{U}(\lambda)$. Then we can write

$$
-z\left(\frac{z}{f(z)}\right)^{\prime}+\frac{z}{f(z)}=\left(\frac{z}{f(z)}\right)^{2} f^{\prime}(z)=1+\lambda w(z)
$$

where $w$ is the Schwarz function with an additional condition $w^{\prime}(0)=0$. We observe from the Schwarz lemma that $|w(z)| \leq|z|^{2}$. As usual it follows that

$$
\frac{z}{f(z)}=1-a_{2} z-\lambda \int_{0}^{1} \frac{w(t z)}{t^{2}} d t, \quad a_{2}=\frac{f^{\prime \prime}(0)}{2 !},
$$

and therefore, by (2.4), we see that

$$
\frac{z f^{\prime}(z)}{f(z)}=\frac{1+\lambda w(z)}{1-a_{2} z-\lambda \int_{0}^{1} \frac{w(t z)}{t^{2}} d t} .
$$


Thus,

$$
\frac{1}{1-\delta}\left(\frac{z f^{\prime}(z)}{f(z)}-\delta\right)=\frac{1+\frac{\lambda w(z)}{1-\delta}+\frac{\delta}{1-\delta}\left[a_{2} z+\lambda \int_{0}^{1} \frac{w(t z)}{t^{2}} d t\right]}{1-a_{2} z-\lambda \int_{0}^{1} \frac{w(t z)}{t^{2}} d t}
$$

Now, $\operatorname{Re}\left(z f^{\prime}(z) / f(z)\right)>\delta$ is equivalent to the condition

$$
\frac{1+\frac{\lambda w(z)}{1-\delta}+\frac{\delta}{1-\delta}\left[a_{2} z+\lambda \int_{0}^{1} \frac{w(t z)}{t^{2}} d t\right]}{1-a_{2} z-\lambda \int_{0}^{1} \frac{w(t z)}{t^{2}} d t} \neq-i T \quad \text { for all } T \in \mathbb{R} \text { and } z \in \Delta,
$$

which is equivalent to

$\lambda\left[\frac{w(z)+(\delta-i(1-\delta) T) \int_{0}^{1} \frac{w(t z)}{t^{2}} d t}{(1-\delta)(1+i T)+a_{2} z(\delta-i T(1-\delta))}\right] \neq-1 \quad$ for all $T \in \mathbb{R}$ and $z \in \Delta$.

If we let

$$
M=\sup _{z \in \Delta, w \in \mathcal{B}, T \in \mathbb{R}}\left|\frac{w(z)+(\delta-i(1-\delta) T) \int_{0}^{1} \frac{w(t z)}{t^{2}} d t}{(1-\delta)(1+i T)+a_{2} z(\delta-i T(1-\delta))}\right|,
$$

then, in view of the rotation invariance of the space $\mathcal{B}$, we obtain

$$
\operatorname{Re}\left(\frac{z f^{\prime}(z)}{f(z)}\right)>\delta \quad \text { if } \lambda M \leq 1 .
$$

This observation shows that it suffices to find $M$. First we notice that

$$
M \leq \sup _{T \in \mathbb{R}}\left\{\frac{1+\sqrt{\delta^{2}+(1-\delta)^{2} T^{2}}}{\left|(1-\delta) \sqrt{1+T^{2}}-a \sqrt{\delta^{2}+(1-\delta)^{2} T^{2}}\right|}\right\},
$$

where, for convenience, we have set $a=\left|a_{2}\right|$. Define $\phi:[0, \infty) \rightarrow \mathbb{R}$ by

$$
\phi(x)=\frac{1+\sqrt{\delta^{2}+(1-\delta)^{2} x}}{(1-\delta) \sqrt{1+x}-a \sqrt{\delta^{2}+(1-\delta)^{2} x}} .
$$

Observe that the denominator in the expression of $\phi(x)$ is positive for all $x \in[0, \infty)$ provided $0 \leq \delta<1 /(1+a)$ and $0 \leq a \leq 1$. Further, it is a simple exercise to see that

$$
\phi^{\prime}(x)=\frac{(1-\delta) N(x)}{2\left[(1-\delta) \sqrt{1+x}-a \sqrt{\delta^{2}+(1-\delta)^{2} x}\right]^{2} \sqrt{1+x} \sqrt{\delta^{2}+(1-\delta)^{2} x}},
$$

where $N(x)=1-2 \delta-\sqrt{\delta^{2}+(1-\delta)^{2} x}+a(1-\delta) \sqrt{1+x}$.

CASE (I): Let $a=0$. Then

$$
\phi^{\prime}(x)=\frac{1-2 \delta-\sqrt{\delta^{2}+(1-\delta)^{2} x}}{2(1-\delta) \sqrt{(1+x)^{3}} \sqrt{\delta^{2}+\left(1-\delta^{2}\right)^{2} x}} .
$$


For $\delta \geq 1 / 3$, we note that $\phi^{\prime}(x) \leq 0$ for all $x \geq 0$ and therefore

$$
\phi(x) \leq \phi(0)=\frac{1+\delta}{1-\delta} .
$$

If $0 \leq \delta<1 / 3$, then $x_{0}=(1-3 \delta) /(1-\delta)$ is the only critical point and $\phi^{\prime \prime}\left(x_{0}\right)<0$. This shows that for $0<\delta<1 / 3, \phi$ attains its maximum value at $x_{0}$ so that

$$
\phi\left(x_{0}\right)=\sqrt{\frac{2(1-\delta)}{1-2 \delta}} .
$$

This gives essentially a direct proof for Corollary 1.3.

CASE (II): Now we consider the case $a \neq 0$. In this case, we have several subcases. Firstly, we let $1 / 2 \leq \delta<1 /(1+a)$. It follows that

$$
N(x) \leq 1-2 \delta \leq 0
$$

because $a(1-\delta) \sqrt{1+x} \leq \sqrt{\delta^{2}+(1-\delta)^{2} x}$. Indeed, the last inequality follows from the fact that $a \leq 1$,

$$
0 \geq(1-\delta)^{2}-\delta^{2}=1-2 \delta \geq a^{2}(1-\delta)^{2}-\delta^{2}
$$

and

$$
x(1-\delta)^{2}\left(1-a^{2}\right) \geq a^{2}(1-\delta)^{2}-\delta^{2} .
$$

Thus, $\phi^{\prime}(x) \leq 0$ for all $x \geq 0$ whenever $1 / 2 \leq \delta<1 /(1+a)$. Next, we consider the case

$$
\frac{1+a}{3+a} \leq \delta<1 / 2
$$

In this case, it suffices to compute

$$
N^{\prime}(x)=-\frac{(1-\delta)^{2}}{2 \sqrt{\delta^{2}+(1-\delta)^{2} x}}+\frac{a(1-\delta)}{2 \sqrt{1+x}}
$$

and note that $N^{\prime}(x) \leq 0$ for $x \geq 0$ if and only if

$$
x(1-\delta)^{2}\left(1-a^{2}\right) \geq a^{2} \delta^{2}-(1-\delta)^{2} .
$$

Since $\delta<1 / 2$ implies that $0>2 \delta-1=\delta^{2}-(1-\delta)^{2} \geq a^{2} \delta^{2}-(1-\delta)^{2}$, the function $N(x)$ is decreasing for $x \geq 0$. Therefore, for $\frac{1+a}{3+a} \leq \delta<1 / 2$, we have

$$
N(x) \leq N(0)=1-2 \delta-\delta+a(1-\delta) \leq 0 \quad \text { for } x \geq 0 .
$$

The above observation shows that $\phi(x)$ defined by (2.5) is a decreasing function on $[0, \infty)$ whenever $\frac{1+a}{3+a} \leq \delta<\frac{1}{1+a}$. In particular,

$$
\phi(x) \leq \phi(0)=\frac{1+\delta}{1-\delta-a \delta} \quad \text { for } \quad \frac{1+a}{3+a} \leq \delta<\frac{1}{1+a} .
$$

CASE (III): Assume $a \neq 0$ and $0 \leq \delta<\frac{1+a}{3+a}$. We make the substitution

$$
t=\frac{1}{\sqrt{\delta^{2}+(1-\delta)^{2} x}}
$$


and note that $\sup _{x \in[0, \infty)} \phi(x)=\sup _{t \in(0,1 / \delta]} \psi(t)$, where $\phi(x)$ becomes

$$
\psi(t)=\frac{1+t}{\sqrt{1+(1-2 \delta) t^{2}}-a}
$$

with the above substitution. Now we compute

$$
\psi^{\prime}(t)=\frac{R(t)}{\left[\sqrt{1+(1-2 \delta) t^{2}}-a\right]^{2} \sqrt{1+(1-2 \delta) t^{2}}},
$$

where $R(t)=1-(1-2 \delta) t-a \sqrt{1+(1-2 \delta) t^{2}}$. Since $R(t)$ decreases,

$$
R(0)=1-a \geq 0>R(1 / \delta)=\frac{3+a}{\delta}\left[\delta-\frac{1+a}{3+a}\right],
$$

and $R(t) \neq 0$ for $t>1 /(1-2 \delta)$, we get the estimate

$$
M \leq\{\psi(t): 0 \leq t \leq 1 /(1-2 \delta), R(t)=0\}=\psi(s),
$$

where

$$
s=\frac{2 \delta-1+a \sqrt{(1-2 \delta)\left(2-a^{2}-2 \delta\right)}}{(1-2 \delta)\left(a^{2}-1+2 \delta\right)} .
$$

A simple calculation shows that

$$
\frac{1}{\psi(s)}=\frac{\sqrt{(1-2 \delta)\left(2-a^{2}-2 \delta\right)}-a(1-2 \delta)}{2(1-\delta)},
$$

a desired result.

Recall the following lemma from [3] which is required for proving Theorem 1.7.

LEMma 2.2. Let $\phi(z)=1+\sum_{n=1}^{\infty} b_{n} z^{n}$ be a nonvanishing analytic function in $\Delta$ and $f(z)=z / \phi(z)$ and $0<\lambda \leq 1$. If any one of the following coefficient conditions holds:

(i) $\sum_{n=2}^{\infty}(n-1)\left|b_{n}\right| \leq \lambda$,

(ii) $\sum_{n=2}^{\infty} n(n-1)\left|b_{n}\right| \leq 2 \lambda$,

then $f \in \mathcal{U}(\lambda)$.

Proof of Theorem 1.5. Assume that $f \in \mathcal{A}$ with $f^{\prime \prime}(0)=0$ and satisfies the condition (1.2), i.e.

$$
\left|2\left(\frac{z}{f(z)}\right)^{2} f^{\prime}(z)\left(1+\frac{z f^{\prime \prime}(z)}{2 f^{\prime}(z)}-\frac{z f^{\prime}(z)}{f(z)}\right)\right| \equiv\left|z^{2}\left(\frac{z}{f(z)}\right)^{\prime \prime}\right|<2 \lambda, \quad z \in \Delta .
$$

We know that (2.6) implies $f \in \mathcal{U}(\lambda)$, which in turn, by Lemma 2.1, gives that $f \in \mathcal{S}^{*}(\delta)$, where $\delta$ is as in Corollary 1.3. Now, the proof may be 
completed by applying Lemma 2.1. To do this, we let $g(z)=\left(\frac{z}{f(z)}\right)^{2} f^{\prime}(z), \quad \phi(z)=\frac{z f^{\prime}(z)}{f(z)}, \quad p(z)=\left(\frac{z f^{\prime \prime}(z)}{f^{\prime}(z)}+1-\beta\right) \frac{1}{1-\beta}$. Then (2.6) is equivalent to $|g(z)(\beta+(1-\beta) p(z)+1-2 \phi(z))|<\lambda$, which is the same as (2.1) with $\alpha=0$. By Lemma 2.1, we have $\operatorname{Re} p(z)>0$ for $z \in \Delta$ with

$$
\beta=2 \delta-1-\frac{2 \lambda}{1-\lambda} \text {. }
$$

Substituting the values of $\delta$ from (1.1) we get the desired conclusion.

Proof of Theorem 1.7. Let $f \in \mathcal{A}$ be of the form

$$
f(z)=\frac{z}{1+\sum_{k=1}^{\infty} b_{k} z^{k}}
$$

where the denominator is nonvanishing on $\Delta$. Then a simple calculation shows that

$$
\begin{aligned}
2\left(\frac{z}{f(z)}\right)^{2} f^{\prime}(z)\left[1+\frac{1}{2} \frac{z f^{\prime \prime}(z)}{f^{\prime}(z)}-\frac{z f^{\prime}(z)}{f(z)}\right] & =-z\left\{z^{2}\left(\frac{1}{f(z)}-\frac{1}{z}\right)^{\prime}\right\}^{\prime} \\
& =-\sum_{n=2}^{\infty} n(n-1) b_{n} z^{n}
\end{aligned}
$$

and therefore, the given coefficient condition (1.5) implies that

$$
\left|\left(\frac{z}{f(z)}\right)^{2} f^{\prime}(z)\left(1+\frac{1}{2} \frac{z f^{\prime \prime}(z)}{f^{\prime}(z)}-\frac{z f^{\prime}(z)}{f(z)}\right)\right|<\lambda, \quad z \in \Delta .
$$

Further, by Lemma 2.2(ii) and (2.7), the coefficient condition (1.5) implies that $f \in \mathcal{U}(\lambda)$. The desired conclusion follows from Theorem 1.5.

Proof of Theorem 1.8. Define

$$
\left(\frac{z}{f(z)}\right)^{\mu+1} f^{\prime}(z)=p(z)=1+a_{2}(1-\mu) z+\frac{(2-\mu)\left(2 a_{3}-(\mu+1) a_{2}^{2}\right) z^{2}}{2}+\cdots .
$$

Then $p$ is analytic in $\Delta, p(0)=1$ and $p(z) \neq 0$ in $\Delta$. Logarithmic derivative of the last equation shows that

$$
(\mu+1)\left(1-\frac{z f^{\prime}(z)}{f(z)}\right)+\frac{z f^{\prime \prime}(z)}{f^{\prime}(z)}=\frac{z p^{\prime}(z)}{p(z)}, \quad z \in \Delta,
$$

and therefore, the given condition (1.6) is equivalent to

$$
\left|\frac{z p^{\prime}(z)}{p(z)}\right|<\log (1+\lambda), \quad z \in \Delta .
$$

We can write

$$
\frac{z p^{\prime}(z)}{p(z)}=[\log (1+\lambda)] w(z),
$$


where $w \in \mathcal{B}:=\{w \in \mathcal{H}: w(0)=0,|w(z)|<1$ for $z \in \Delta\}$. Therefore

$$
\int_{0}^{z} \frac{p^{\prime}(t)}{p(t)} d t=\log (1+\lambda) \int_{0}^{z} \frac{w(t)}{t} d t, \quad z \in \Delta
$$

from which we get

so that

$$
\log p(z)=\log (1+\lambda) \int_{0}^{1} \frac{w(t z)}{t} d t
$$

$$
p(z)=\exp \left[\log (1+\lambda) \int_{0}^{1} \frac{w(t z)}{t} d t\right]
$$

Thus,

$$
|p(z)-1| \leq \exp \left(\log (1+\lambda)\left|\int_{0}^{1} \frac{w(t z)}{t} d t\right|\right)-1<\lambda, \quad z \in \Delta .
$$

The desired conclusion follows.

For the proof of (ii), because $f^{\prime \prime}(0)=0$, it suffices to observe that $w \in \mathcal{B}$ with $w^{\prime}(0)=0$ so that, by the Schwarz lemma, $|w(z)| \leq|z|^{2}$ for $z \in \Delta$. The desired conclusion follows if we apply this inequality to the last inequality.

3. Two conjectures. The results of this paper (e.g. Theorem 1.5) motivate the following

Conjecture 1. If $0<\lambda \leq 3-2 \sqrt{2}$ then each $f \in \mathcal{U}(\lambda)$ with $f^{\prime \prime}(0)=0$ is convex in $\Delta$.

We recall that

- $f \in \mathcal{U}(\lambda), f^{\prime \prime}(0)=0$ and $0<\lambda \leq 1 / 3 \Rightarrow f \in \mathcal{S}^{*}(1 / 2)$,

- $f \in \mathcal{P}(2 \lambda), f^{\prime \prime}(0)=0$ and $0<\lambda \leq 3-2 \sqrt{2} \Rightarrow f \in \mathcal{K}$.

We also observe $1 / 3>3-2 \sqrt{2}$ which is expected, since $\mathcal{K} \subsetneq \mathcal{S}^{*}(1 / 2)$ and $\mathcal{P}(2 \lambda) \subset \mathcal{U}(\lambda)$. Further, the method of proof of Theorem 1.2 suggests the following which we are unable to settle at present.

Conjecture 2. The $\lambda(\delta)$ given in Theorem 1.2 is sharp for $f \in \mathcal{U}(\lambda)$ to be starlike of order $\delta$.

\section{References}

[1] P. L. Duren, Univalent Functions, Grundlehren Math. Wiss. 259, Springer, New York, 1983.

[2] A. W. Goodman, Univalent Functions, Vols. 1-2, Mariner, Tampa, FL, 1983.

[3] M. Obradović and S. Ponnusamy, New criteria and distortion theorems for univalent functions, Complex Var. Theory Appl. 44 (2001), 173-191. (Also Reports of the Department of Mathematics, Preprint 190, June 1998, University of Helsinki). 
[4] M. Obradović, S. Ponnusamy, V. Singh and P. Vasundhra, Univalency, starlikeness and convexity applied to certain classes of rational functions, Analysis (Munich) 22 (2002), 225-242.

[5] M. Obradović and N. Tuneski, On certain properties of a class of univalent functions, Filomat 13 (1999), 59-65.

[6] S. Ozaki and M. Nunokawa, The Schwarzian derivative and univalent functions, Proc. Amer. Math. Soc. 33 (1972), 392-394.

[7] M. O. Reade, H. Silverman and P. G. Todorov, Classes of rational functions, in: Contemp. Math. 38, Amer. Math. Soc., 1985, 99-103.

Department of Mathematics

Indian Institute of Technology

IIT-Madras

Chennai 600 036, India

E-mail: samy@iitm.ac.in

Reçu par la Rédaction le 18.11.2003

Révisé le 22.2.2005 